'Departamento de Nutrición, Facultad de Medicina,

Universidad de Chile. Santiago, Chile.

${ }^{a}$ Médico Veterinaria. Magíster en Nutrición y Alimentos. Instituto de Nutrición y Tecnología de los Alimentos (INTA), Universidad de Chile.

bBiotecnólogo. Doctor en Fisiología y Alimentación (PhD). 'Bioquímico. Doctor en Ciencias Biológicas (PhD).

Financiado por FONDECYT 1130240.

Conflictos de intereses: ninguno que declarar.

Recibido el 9 de diciembre de 2014, aceptado el 31 de marzo de 2015.

Correspondencia a: Francisco Pérez-Bravo. Av. Independencia 1027, Departamento de Nutrición, Bloque K, Santiago.

Teléfono: + 569-90324056 fperez@med.uchile.cl

\section{La metilación global del ADN y los niveles de homocisteína en plasma se encuentran disminuidos en pacientes con diabetes mellitus tipo 1}

\author{
VIVIANA ARROYO-JOUSSE ${ }^{1, a}$, DIEGO F. GARCÍA-DÍAZ ${ }^{1, b}$, \\ FRANCISCO PÉREZ-BRAVO ${ }^{1, c}$
}

\section{Global DNA methylation and homocysteine levels are lower in type 1 diabetes patients}

Background: The worldwide rise in the incidence of Type 1 Diabetes (T1D), and the concordance rate between monozygotic twins (50\%), indicate a strong effect of the environment as an underlying factor of this disease. This process can occur throughout epigenetic modifications of gene expression such as DNA methylation, in which several nutrients participate as cofactors. Aim: To determine DNA methylation status in T1D patients and if it is related to plasma levels of folates and homocysteine (Hcy). Material and Methods: We obtained blood samples from 25 T1D patients aged $13.7 \pm 5.9$ years (11 males) and 25 healthy subjects aged $31.1 \pm 7.8$ years ( 16 males). DNA methylation was measured using a colorimetric kit in extracted DNA. Results are expressed as median (interquartile range). Results: Compared with healthy controls, T1D patients had lower global DNA methylation (0.85 (0.91)\% and $1.25(1.16) \%$ respectively, $p<0.02)$ and Hcy levels $(4.8(1.1) \mu \mathrm{mol} / \mathrm{L}$ and $7.3(1.4) \mu \mathrm{mol} / \mathrm{L}$ respectively $p<0.01)$. There were no differences in folate levels between groups. A significant association between folates and global DNA methylation status was observed in T1D patients $(r=-0.564, p<0.01)$ and healthy subjects $(r=0.440$, $p=0.03)$. Conclusions: TD1 patients had lower levels of Hcy and global DNA methylation. It is relevant to further investigate if this imbalance also induces epigenetic changes in a gene-specific manner, especially in key genes involved in T1D pathogenesis.

(Rev Med Chile 2015; 143: 562-568)

Key words: DNA methylation; Epigenetics; Folate; Type 1 Diabetes.
L a diabetes mellitus tipo 1 (DM1) se ha definido como una enfermedad autoinmune crónica, que tiene como resultado la destrucción parcial o completa de las células $\beta$ pancreáticas lo que se traduce finalmente en la presentación de la triada clásica de síntomas (polidipsia, polifagia, poliuria), acompañada de hiperglicemia y pérdida inexplicable de peso. Es comúnmente diagnosticada en niños, adolescentes y adultos jóvenes ${ }^{1-4}$.
La incidencia de esta patología ha aumentado en las últimas décadas tanto en Chile ${ }^{5,6}$ como a nivel mundial ${ }^{7}$. Este aumento, sin embargo, no se ha acompañado de una mayor frecuencia en los genotipos que confieren mayor riesgo, sino más bien de una disminución de ellos ${ }^{8}$. En este sentido, existen alrededor de 40 loci que modifican el riesgo de contraer $\mathrm{DM} 1^{9}$, lo que la convierte en una enfermedad de carácter poligénica. No obstante, la tasa de concordancia entre gemelos 
monocigotos es de alrededor de $50 \%{ }^{10,11}$, lo que sugiere el fuerte impacto de factores ambientales sobre la incidencia de la enfermedad ${ }^{12}$.

El ambiente es capaz de modular la expresión génica mediante modificaciones epigenéticas, las que se definen como cambios heredables que no involucran alteraciones en la secuencia del $\mathrm{ADN}^{13,14}$. Entre ellas se encuentra la metilación del ADN, proceso esencial en la regulación de la expresión génica en mamíferos. Este mecanismo involucra la adición covalente de un grupo metilo $\left(\mathrm{CH}_{3}\right)$, a la citosina de un dinucleótido citosina-guanina $(\mathrm{CpG})$, desde el donante de metilos S-Adenosil Metionina (SAMe). Esta reacción es catalizada por las enzimas ADN metiltransferasas (DNMTs) y ocurre tanto a nivel global como a nivel gen-específico. Así, cuando regiones ricas en estos dinucleótidos $\mathrm{CpG}$, están ubicadas en la región promotora de los genes, su metilación conduce al silenciamiento de dicho gen ${ }^{14-18}$.

En un estudio realizado el año $2011^{19}$ se identificaron 132 posiciones de metilación variables entre gemelos monocigotos discordantes (uno diabético tipo 1 diagnosticado antes de los 20 años y el otro sano). Los autores demostraron, además, que estas posiciones variables se detectan antes del diagnóstico clínico, en normoglicemia, y son independientes del proceso patológico, de la disfunción metabólica y del tratamiento con insulina. Disanto y cols. ${ }^{20}$, encontraron un patrón diferencial de metilación en el ADN de cuatrillizos monocigotos discordantes (2 diabéticos tipo 1, 1 pre-diabético y 1 sano). De igual forma, Stefan y cols. ${ }^{21}$, demostraron que el patrón de metilación en gemelos concordantes para DM1 presenta más similitudes que el patrón de metilación entre gemelos discordantes. Estos estudios sugieren que variaciones en la metilación del ADN podrían jugar un papel importante en la patogénesis y la etiología de enfermedades complejas como la DM1.

Diversos nutrientes (folatos, vitamina B2, B6, B12, colina y betaína), actúan como cofactores o participan directamente en la vía de generación de SAMe. La homocisteína (Hcy) es un aminoácido azufrado y un intermediario relevante en el ciclo de las moléculas de un carbono. Su principal vía de metabolización, a través de su remetilación, da como resultado la síntesis de metionina la cual, posteriormente, se convierte en SAMe. Esta vía usa folato como substrato y vitamina B12 como cofactor. Su otra vía de metabolización es mediante su transulfuración que resulta en la producción de cisteína y que requiere de vitamina B6 como cofactor $^{22}$.

Bajo condiciones de déficit de folatos, disminuye la disponibilidad de grupos de un carbono requeridos para las reacciones de metilación; esto conduce a la acumulación de Hcy. La hiperhomocisteinemia (hHcy) se traduce en la acumulación de S-Adenosil Homocisteína (SAH) que inhibe a las enzimas metiltransferasas dependientes de SAMe, incluyendo a las DNMTs, lo que eventualmente podría llevar a un estado de hipometilación global del $\mathrm{ADN}^{23,24}$.

Por lo tanto, el objetivo de este trabajo es determinar el estatus de metilación global en diabéticos tipo 1 y comparar dicho estatus con controles sanos, de manera de dilucidar si la regulación epigenética se encuentra alterada en la DM1. Además se evaluará si el estatus de metilación se asocia con los niveles plasmáticos de folatos y homocisteína.

\section{Material y Método}

Se estudiaron 25 pacientes con DM1 (1-25 años) y 25 controles ( $>20$ años), sin DM1, enfermedades autoinmunes, ni antecedentes familiares directos de dichas patologías. Se obtuvieron muestras de sangre, en ayunas, las que fueron procesadas antes de una hora. Los pacientes fueron reclutados del Instituto de Investigación Materno Infantil (IDIMI), de la Universidad de Chile, en el Hospital San Borja Arriarán. Todos los individuos reclutados en este estudio firmaron un consentimiento informado; en el caso de niños menores de 7 años éste fue firmado por sus padres o tutores. Este estudio fue aprobado por el Comité de Ética de la Facultad de Medicina de la Universidad de Chile y por el Comité de Ética del Servicio de Salud Central (Hospital San Borja Arriarán).

Para la extracción del ADN se utilizó un kit comercial (Wizard Genomic DNA Purification Kit, Promega ${ }^{\circledR}$, Wisconsin, U.S.A.). Los niveles de folatos y de Hcy fueron determinados en el laboratorio clínico RedLab (Santiago, Chile) en suero y plasma, respectivamente. El porcentaje de metilación global se midió con el kit Methyl Flash Methylated DNA Quantification kit (Colorimetric) (Epigentek ${ }^{\circledR}$, Nueva York, U.S.A.) de acuerdo a lo descrito por el fabricante. 
Se detectó en plasma, de pacientes y controles, la presencia de auto anticuerpos contra los antígenos $\beta$ pancreáticos: ácido L-glutámico decarboxilasa (anti-GAD65), antígeno de insulinoma 2 (anti-IA2) y contra la molécula transportadora de Zinc 8 (anti-ZnT8), con kits de ELISA comerciales específicos para cada uno de ellos (Medizym, Eagle Biosciences $^{\circledR}$, Boston, U.S.A.).

Se comprobó la distribución normal de las variables utilizando el test de Shapiro-Wilk. La comparación entre grupos se realizó mediante la prueba U de Mann Whitney. Se utilizó el test de Spearman para determinar asociación entre las variables. Los resultados son expresados como mediana (rango intercuartil). Para los análisis estadísticos se consideró un nivel de significancia $\mathrm{p}<0,05$. Los datos fueron analizados con el Software estadístico Graph Pad Prism 6.0.

\section{Resultados}

En la Tabla 1 se encuentran resumidos los datos clínicos de los individuos en estudio. No se detectaron auto-anticuerpos contra antígenos pancreáticos en el suero de los controles, mientras que en los pacientes $66,7 \%$ resultó positivo para anti-GAD65 y anti-IA2 y 42,9\% de los pacientes presentó anticuerpos anti-ZnT8; sólo dos pacientes fueron negativos para los 3 anticuerpos.

El porcentaje de metilación global (Figura 1A) se encontró más bajo en pacientes diabéticos
$(0,85 \%[0,91])$ que en controles $(1,25 \%[1,16])$ $(\mathrm{p}=0,015)$ y se observó que el estatus de metilación global aumenta con la edad $(\mathrm{p}=0,005)$ (Figura 1B) en DM1, pero no en controles. De igual forma, dentro del grupo de pacientes, los niveles de Hcy fueron más altos en adolescentes mayores de 15 años que en niños entre 10-15 años $(\mathrm{p}=0,032)$ y más altos que en pacientes menores de 10 años $(\mathrm{p}=0,032)$.

En cuanto a los niveles de folatos séricos no se encontraron diferencias entre pacientes $y$ controles $(\mathrm{p}=0,357)$, mientras que los niveles plasmáticos de Hcy (Figura 2) fueron menores en pacientes $(4,8 \mu \mathrm{mol} / \mathrm{L}[1,1])$ que en controles $(7,3$ $\mu \mathrm{mol} / \mathrm{L}[1,4])(\mathrm{p}<0,0001)$. Los niveles de folatos

Tabla 1. Características clínicas de los grupos en estudio

\begin{tabular}{|lcc|}
\hline Características clínicas & DM1 & Controles \\
$\mathrm{n}$ & 25 & 25 \\
Edad (años) & $13,7 \pm 5,9$ & $31,1 \pm 7,8$ \\
Sexo & $\mathrm{F}=14$ & $\mathrm{~F}=9$ \\
& $\mathrm{M}=11$ & $\mathrm{M}=16$ \\
Tiempo desde debut (años) & $7 \pm 5,7$ & - \\
HbA1c (\%) & $8,94 \pm 1,69$ & - \\
Anti-GAD (\% presencia) & 66,7 & 0 \\
Anti-IA2 (\% presencia) & 66,7 & 0 \\
Anti-ZnT8 (\% presencia) & 42,9 & 0 \\
\hline
\end{tabular}

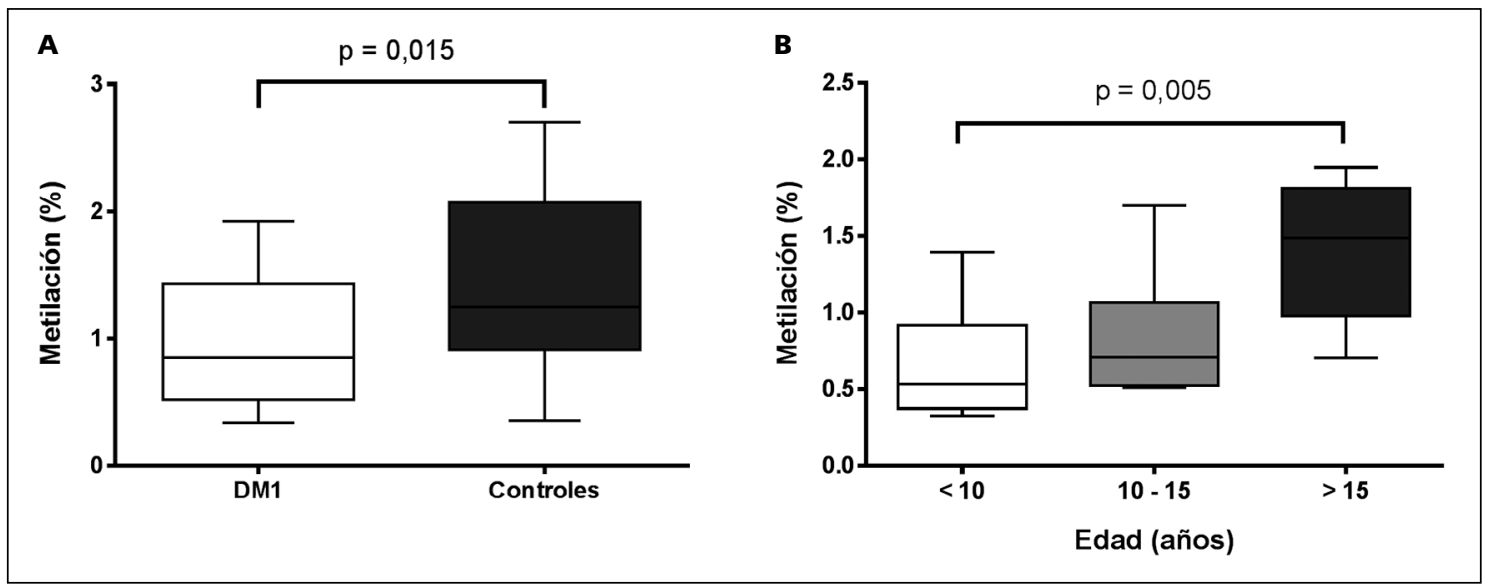

Figura 1. Estatus de Metilación global en pacientes diabéticos tipo 1 y controles. A: Porcentaje de metilación global del ADN entre pacientes $(n=25)$ y controles $(n=25)$. B: Porcentaje de metilación global en DM1 de acuerdo a la edad. $<10$ años $(\mathrm{n}=9) ; 10-15$ años $(\mathrm{n}=8) ;>15$ años $(\mathrm{n}=8)$. DM1: Diabetes Mellitus tipo 1 . 
se correlacionaron de forma lineal con los niveles de metilación global tanto en DM1 $(\mathrm{r}=-0,564$; $\mathrm{p}=0,003)$ como en controles $(\mathrm{r}=0,440 ; \mathrm{p}=0,028)$ (Figura 3), no observándose asociación alguna entre metilación global y Hcy.

\section{Discusión}

Las modificaciones epigenéticas son heredables y son influenciadas por factores ambientales, es por ello que el objetivo de este estudio fue evidenciar diferencias en el patrón de metilación global entre pacientes diabéticos e individuos sanos, encontrándose menores niveles de meti-

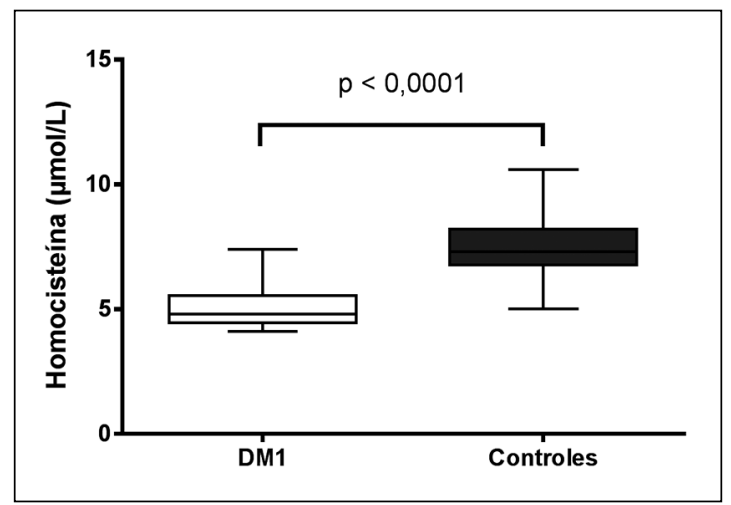

Figura 2. Homocisteína plasmática. Niveles plasmáticos de homocisteína en pacientes $(n=25)$ y en controles $(n=24)$. DM1: Diabetes Mellitus tipo 1. lación global en DM1 ( $\mathrm{p}=0,015)$. La formación de SAMe, el principal donante de grupos metilo para las reacciones de metilación del organismo, ocurre en el llamado ciclo de las moléculas de un carbono, en el que participan diversos nutrientes como cofactores o intermediarios ${ }^{14}$, incluyendo al aminoácido sulfurado metionina. La Hcy, juega un papel central en el metabolismo de la metionina y por tanto en el ciclo de las moléculas de un carbono. Existen dos vías independientes de remetilación de la Hcy: la primera catalizada por la enzima metionina sintetasa (MS) y la segunda por la betaína: homocisteína metiltransferasa (BHMT); ambas conducen a la formación de metionina. Por otro lado, la Hcy puede ser irreversiblemente convertida en cisteína, mediante la vía de transulfuración, reacción catalizada por las enzimas cistationina- $\beta$-sintasa (CBS) y cisteína$\gamma$-liasa (CGL) ${ }^{25,26}$.

El metabolismo de la Hcy-Metionina se encuentra alterado en la DM1, ya que los niveles de insulina son capaces de modular la actividad de las enzimas participantes en el ciclo. Esto fue demostrado en ratas (con diabetes inducida por tratamiento con estreptozotocina), en donde se evidenció una mayor actividad de las enzimas CBS y CGL, lo que se revierte con el tratamiento con insulina exógena ${ }^{27}$, en cultivos celulares de hepatocitos ${ }^{28}$ y en humanos con DM1 en donde se observó una mayor actividad de la vía de transulfuración en pacientes sin tratamiento con insulina ${ }^{29}$. Estos estudios concuerdan con lo observado por

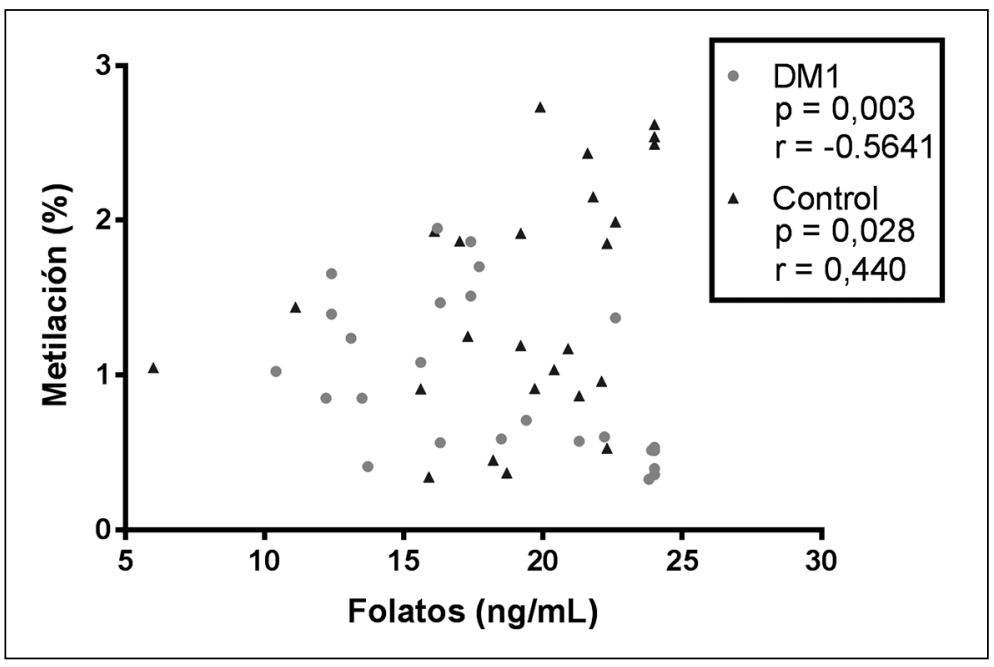

Figura 3. Niveles de Folatos y Metilación del ADN. Se describe una asociación negativa entre niveles de folatos y la metilación global del ADN en pacientes (círculos) y una asociación positiva entre niveles de folatos y metilación global del ADN en controles (triángulos). DM1: Diabetes Mellitus tipo 1; $r$, coeficiente de correlación de Spearman. 
Williams et al. ${ }^{30}$, quienes además de una mayor actividad en las enzimas de la transulfuración encontraron una mayor actividad hepática de BHMT y una menor actividad de MS. Junto con esto evidenciaron, a nivel hepático, un estatus disminuido de metilación global en ratas diabéticas. Además de la insulina, se ha demostrado que niveles elevados de glucagón también aumentan la actividad de CBS y CGL, potenciando entonces la vía de transulfuración de la $\mathrm{Hcy}^{31}$.

En todos los trabajos recién descritos se observaron menores niveles de Hcy en DM1 con respecto a controles, lo que concuerda con nuestros resultados. Estos menores niveles de Hcy en pacientes podrían estar evidenciando una alteración en el metabolismo de la metionina, y por tanto en el ciclo de un carbono, determinando así una menor disponibilidad de SAMe y por tanto un menor estatus de metilación global, tal como se evidenció en este estudio.

Se ha demostrado que la hHcy en niños y adolescentes con DM1, sin complicaciones, es una característica poco frecuente ${ }^{32,33}$ y que los bajos niveles observados en estos grupos etarios se deben a una mayor tasa de filtración glomerular ${ }^{34}$, siendo ésta especialmente común en los primeros 5 años de duración de esta patología ${ }^{35}$. La hHcy comienza a aparecer en concordancia con la presentación de alteraciones en la función renal u otras complicaciones ${ }^{36-40}$.

No encontramos asociación entre la HbAlc (hemoglobina glicosilada) con la metilación global ni con los niveles de Hcy en nuestro pacientes. Sin embargo, es importante destacar que sólo dos de ellos presentaron niveles de HbAlc menores a $7 \%$, por lo que el control metabólico en estos pacientes no es óptimo, lo que podría influenciar lo observado en este trabajo.

Existen diversos estudios que han demostrado una relación entre el estatus nutricional de folatos y el estatus de metilación en humanos, tema que ha sido revisado previamente ${ }^{17}$, y que en este trabajo se evidencia por la presencia de correlación entre ambos parámetros, tanto en DM1 como en controles, destacando el rol de la nutrición como modulador de mecanismos epigenéticos. La asociación negativa entre folatos y metilación global en pacientes podría deberse a la alteración en el metabolismo de la Hcy más que a un efecto directo de los niveles de folatos en este grupo, los cuales fueron similares a los niveles en controles. Por lo mismo, en controles se observa una asociación positiva entre folatos y metilación global, la que concuerda con los mayores niveles de metilación global junto con niveles más altos de Hcy, en estos individuos.

Este trabajo tiene ciertas limitaciones, entre ellas la falta de concordancia entre las edades del grupo de pacientes y el grupo de controles. Aun cuando en el grupo control no se observó diferencias por edad en los niveles de Hcy y de metilación global, en individuos de mayor edad los patrones de metilación pudieran variar en mayor grado que en individuos más jóvenes.

En este estudio hemos evidenciado menores niveles de Hcy y un estatus de metilación global disminuido en pacientes diabéticos tipo 1 , lo que concuerda con otros estudios que han demostrado una alteración en el metabolismo de la homocisteína lo que modifica, a su vez, los patrones de metilación del ADN. Es de gran relevancia realizar investigaciones posteriores que permitan dilucidar si esta alteración en la regulación epigenética se da a nivel gen específico, en genes involucrados en el desarrollo de esta patología, y si estos cambios epigenéticos son visibles incluso antes del inicio de la enfermedad. De esta forma se podrá avanzar en la búsqueda de marcadores de riesgo temprano y de mecanismos de prevención durante etapas especialmente susceptibles a modificaciones epigenéticas como la gestación y el período neonatal temprano.

Agradecimientos: Este trabajo ha sido financiado por el proyecto FONDECYT 1130240 (Dr. Francisco Pérez-Bravo). Agradecemos a todos los pacientes participantes en esta investigación y al apoyo del Hospital San Borja Arriarán.

\section{Referencias}

1. Pugliese A. The multiple origins of Type 1 diabetes. Diabet Med 2012; 30 (2): 135-46.

2. Atkinson MA. The pathogenesis and natural history of type 1 diabetes. Cold Spring Harb Perspect Med 2012; 2 (11): 1-18.

3. Canivell S, Gomis R. Diagnosis and classification of autoimmune diabetes mellitus. Autoimmun Rev 2014; 13 (4-5): 403-7.

4. Nokoff N, Rewers M. Pathogenesis of type 1 diabetes: lessons from natural history studies of high-risk individuals. Ann N Y Acad Sci. 2013; 1281: 1-15. 
5. Santos J, Carrasco E, Moore A, Pérez-Bravo F, Albala C. Incidence rate and spatio-temporal clustering of Type 1 Diabetes in Santiago, Chile, from 1997 to 1998. Saúde Pública 2001; 35 (1): 96-100.

6. Carrasco E, Angel B, Codner E, García D, Ugarte F, Bruzzone ME, et al. [Type 1 diabetes mellitus incidence in Santiago, Chile. Analysis by counties in the period 2000-2004]. Rev Med Chile 2006; 134 (10): 1258-64.

7. Adeghate E, Schattner P, Dunn E. An update on the etiology and epidemiology of diabetes mellitus. Ann N Y Acad Sci 2006; 1084: 1-29.

8. Hermann R, Knip M, Veijola R, Simell O, Laine AP, Akerblom HK, et al. Temporal changes in the frequencies of HLA genotypes in patients with Type 1 diabetes-indication of an increased environmental pressure? Diabetologia 2003; 46 (3): 420-5.

9. Barrett JC, Clayton DG, Concannon P, Akolkar B, Cooper JD, Erlich HA, et al. Genome-wide association study and meta-analysis find that over 40 loci affect risk of type 1 diabetes. Nat Genet 2009; 41 (6): 703-7.

10. Redondo MJ, Yu L, Hawa M, Mackenzie T, Pyke DA, Eisenbarth GS, et al. Heterogeneity of type I diabetes: analysis of monozygotic twins in Great Britain and the United States. Diabetologia 2001; 44 (3): 354-62.

11. Hyttinen V, Kaprio J, Kinnunen L, Koskenvuo M, Tuomilehto J. Genetic liability of type 1 diabetes and the onset age among 22,650 young Finnish twin pairs: a nationwide follow-up study. Diabetes 2003; 52 (4): 1052-5.

12. Knip M, Simell O. Environmental triggers of type 1 diabetes. Cold Spring Harb Perspect Med 2012; 2 (7): $1-15$.

13. Hewagama A, Richardson B. The genetics and epigenetics of autoimmune diseases. J Autoimmun 2009; 33 (1): 3-11.

14. Anderson OS, Sant KE, Dolinoy DC. Nutrition and epigenetics: an interplay of dietary methyl donors, one-carbon metabolism and DNA methylation. J Nutr Biochem 2012; 23 (8): 853-9.

15. Stankov K, Benc D, Draskovic D. Genetic and epigenetic factors in etiology of diabetes mellitus type 1. Pediatrics 2013; 132 (6): 1112-22.

16. Dang MN, Buzzetti R, Pozzilli P. Epigenetics in autoimmune diseases with focus on type 1 diabetes. Diabetes Metab Res Rev 2013; 29 (1): 8-18.

17. Ly A, Hoyt L, Crowell J, Kim YI. Folate and DNA methylation. Antioxid Redox Signal 2012; 17 (2): 302-26.

18. Bird A. DNA methylation patterns and epigenetic memory. Genes Dev 2002; 16 (1): 6-21.

19. Rakyan VK, Beyan H, Down TA, Hawa MI, Maslau S, Aden $\mathrm{D}$, et al. Identification of type 1 diabetes-associa- ted DNA methylation variable positions that precede disease diagnosis. PLoS Genet 2011; 7 (9): 1-9.

20. Disanto G, Vcelakova J, Pakpoor J, Elangovan RI, Sumnik Z, Ulmannova T, et al. DNA methylation in monozygotic quadruplets affected by type 1 diabetes. Diabetologia 2013; 56 (9): 2093-5.

21. Stefan M, Zhang W, Concepcion E, Yi Z, Tomer Y. DNA methylation profiles in type 1 diabetes twins point to strong epigenetic effects on etiology. J Autoimmun 2013; 50: 33-7.

22. Saeed BO, Banerjee K, Nixon SJ, Brown K. Plasma homocysteine concentrations in patients with Type 1 diabetes. Diabet Med 2003; 20 (10): 867-8.

23. Al-Maskari MY, Waly MI, Ali A, Al-Shuaibi YS, Ouhtit A. Folate and vitamin B12 deficiency and hyperhomocysteinemia promote oxidative stress in adult type 2 diabetes. Nutrition 2012; 28 (7-8): e23-6.

24. Nazki FH, Sameer AS, Ganaie BA. Folate: metabolism, genes, polymorphisms and the associated diseases. Gene 2014; 533 (1): 11-20.

25. Wijekoon P, Brosnan M, Brosnan J. Homocysteine Metabolism in Diabetes. In: S. K. Cheema, editor. Biochemestry of Atherosclerosis. New York: Springer; 2006.

26. Finkelstein JD. The metabolism of homocysteine: pathways and regulation. Eur J Pediatr 1998; 157 Suppl 2: S40-4.

27. Jacobs RL, House JD, Brosnan ME, Brosnan JT. Effects of streptozotocin-induced diabetes and of insulin treatment on homocysteine metabolism in the rat. Diabetes 1998; 47 (12): 1967-70.

28. Chiang EP, Wang YC, Chen WW, Tang FY. Effects of insulin and glucose on cellular metabolic fluxes in homocysteine transsulfuration, remethylation, S-adenosylmethionine synthesis, and global deoxyribonucleic acid methylation. J Clin Endocrinol Metab 2009; 94 (3): 1017-25.

29. Abu-Lebdeh HS, Barazzoni R, Meek SE, Bigelow ML, Persson XM, Nair KS. Effects of insulin deprivation and treatment on homocysteine metabolism in people with type 1 diabetes. J Clin Endocrinol Metab 2006; 91 (9): 3344-8.

30. Williams KT, Garrow TA, Schalinske KL. Type I diabetes leads to tissue-specific DNA hypomethylation in male rats. J Nutr 2008; 138 (11): 2064-9.

31. Jacobs RL, Stead LM, Brosnan ME, Brosnan JT. Hyperglucagonemia in rats results in decreased plasma homocysteine and increased flux through the transsulfuration pathway in liver. J Biol Chem 2001; 276 (47): 43740-7.

32. Meloni GF, Tonolo GC, Zuppi C, Zappacosta B, Musumeci S. Hyper-homocysteinemia is not a main feature 
of juvenile uncomplicated type 1 diabetes. J Atheroscler Thromb 2005; 12 (1): 14-9.

33. Salardi S, Cacciari E, Sassi S, Grossi G, Mainetti B, Dalla Casa C, et al. Homocysteinemia, serum folate and vitamin B12 in very young patients with diabetes mellitus type 1. J Pediatr Endocrinol Metab 2000; 13 (9): 1621-7.

34. Veldman BA, Vervoort G, Blom H, Smits P. Reduced plasma total homocysteine concentrations in Type 1 diabetes mellitus is determined by increased renal clearance. Diabet Med 2005; 22 (3): 301-5.

35. Elbert A. Enfermedad Renal en Pacientes con Diabetes Mellitus. In: M. García de los Ríos y P. Durruty, editores. Diabetes Mellitus. 3era ed. Santiago: Mediterráneo Ltda.; 2014. p. 227-39.

36. Abdel Aziz MT, Fouad HH, Mohsen GA, Mansour M, Abdel Ghaffar S. TNF-alpha and homocysteine levels in type 1 diabetes mellitus. East Mediterr Health J. 2001; 7
(4-5): 679-88.

37. Saeed BO, Nixon SJ, White AJ, Summerfield GP, Skillen AW, Weaver JU. Fasting homocysteine levels in adults with type 1 diabetes and retinopathy. Clin Chim Acta 2004; 341 (1-2): 27-32.

38. Agullo-Ortuno MT, Albaladejo MD, Parra S, Rodríguez-Manotas M, Fenollar M, Ruiz-Espejo F, et al. Plasmatic homocysteine concentration and its relationship with complications associated to diabetes mellitus. Clin Chim Acta 2002; 326 (1-2): 105-12.

39. Vaccaro O, Perna AF, Mancini FP, Iovine C, Cuomo V, Sacco M, et al. Plasma homocysteine and microvascular complications in type 1 diabetes. Nutr Metab Cardiovasc Dis 2000; 10 (6): 297-304.

40. Tessari P, Coracina A, Kiwanuka E, Vedovato M, Vettore $\mathrm{M}$, Valerio A, et al. Effects of insulin on methionine and homocysteine kinetics in type 2 diabetes with nephropathy. Diabetes 2005; 54 (10): 2968-76. 